\title{
Shenqi fuzheng, an injection concocted from chinese medicinal herbs, combined with platinum-based chemotherapy for advanced non-small cell lung cancer: a systematic review
}

\author{
Ju Dong ${ }^{1+}$, Shi-Yue Su${ }^{2+}$, Min-Yan Wang ${ }^{3+}$, Zhen Zhan ${ }^{4 *}$
}

\begin{abstract}
Background: Platinum-based chemotherapy has been a standard therapy for advanced non-small cell lung cancer (NSCLC), but it has high toxicity. In China, Shenqi Fuzheng, a newly developed injection concocted from Chinese medicinal herbs has been reported that may increase efficacy and reduce toxicity when combined with platinum-based chemotherapy, but little is known about it outside of China. The aim of this study was to systematically review the existing clinical evidence on Shenqi Fuzheng Injection(SFI) combined with platinum-based chemotherapy for advanced NSCLC.

Methods: Pubmed, Cochrane Library, EMBASE, CNKI, and CBM search were organized for all documents published, in English and Chinese, until April 2010. The randomized controlled clinical trials were selected based on specific criteria, in which a SFI plus platinum-based chemotherapy treatment group was compared with a platinum-based chemotherapy control group for patients with advanced NSCLC. The quality of studies was assessed by modified Jadad's scale, and Revman 4.2 software was used for data syntheses and analyses.

Results: Twenty nine studies were included in this review based on our selection criteria. Of them, ten studies were of high quality and the rest were of low quality, according to the modified Jadad scale. The meta-analysis showed there was a statistically significant higher tumor response $(\mathrm{RR}, 1.19 ; 95 \% \mathrm{Cl}, 1.07$ to $1.32 ; \mathrm{P}=0.001)$ and performance status ( $(\mathrm{RR}, 1.57 ; 95 \% \mathrm{Cl}, 1.45$ to $1.70 ; \mathrm{P}<0.00001)$; but lower severe toxicity for $\mathrm{WBC}(\mathrm{RR}, 0.37 ; 95 \% \mathrm{Cl}, 0.29$ to $0.47 ; \mathrm{P}<$ 0.00001), PLT (RR, 0.33; 95\% Cl, 0.21 to $0.52 ; \mathrm{P}<0.00001), \mathrm{HB}(\mathrm{RR}, 0.44 ; 95 \% \mathrm{Cl}, 0.30$ to $0.66 ; \mathrm{P}<0.0001$ ) and nausea and vomiting (RR, $0.32 ; 95 \% \mathrm{Cl}, 0.22$ to $0.47 ; \mathrm{P}<0.00001)$, when the SFI plus platinum-based chemotherapy treatment group was compared with the platinum-based chemotherapy control group. Sensitivity analysis was restricted to studies with the high quality, and the result was similar when the studies with low quality were excluded. Asymmetry was observed in a funnel plot analysis, and Egger's test also indicated an evidence of publication bias $(P=0.016)$.

Conclusions: SFI intervention appears to be useful to increase efficacy and reduce toxicity when combined with platinum-based chemotherapy for advanced NSCLC, although this result needs to be further verified by more highquality trials.
\end{abstract}

\section{Background}

Lung cancer is the leading cause of cancer-related mortality around the world, of which non-small cell lung cancer (NSCLC) accounts for approximately 85\% [1]. Moreover, most NSCLC cases already reach stages III

\footnotetext{
* Correspondence: zhanzheng_nj@163.com

+ Contributed equally

${ }^{4}$ Institute of Basic Medical Science, Nanjing University of Traditional Chinese Medicine, Nanjing, P.R China

Full list of author information is available at the end of the article
}

and IV at the time of diagnosis indicating an advanced and often inoperable stage of NSCLC. Platinum-based chemotherapy has been a standard therapy and is widely accepted for treatment of advanced NSCLC [1,2]. The superiority of platinum-based chemotherapy over nonplatinum-based chemotherapy has been proved by many randomized clinical trials. However, the resulting hematal and gastrointestinal toxicity, such as leukopenia, thrombopenia, nausea, vomiting and so on, have also been 
reported $[3,4]$, which may seriously affect the patient's survival quality and curative effects. So, questions remain on how to best reduce the toxicity and enhance the curative effect of platinum-based chemotherapy.

In China, to reduce the toxicity and enhance the curative effect of platinum-based chemotherapy, many traditional Chinese medicinal herbs have been widely used combined with platinum-based chemotherapy for the treatment of advanced NSCLC, and some researchers $[5,6]$ have found that combining Chinese medicinal herbs with platinum-based chemotherapy for the treatment of advanced NSCLC may improve survival, tumor response, and performance status, as well as reduce chemotherapy toxicity.

Shenqi Fuzheng is a newly developed injection concocted from two kinds of Chinese medicinal herbs: Radix Astragali (root of astragalus; Chinese name: huangqi) and Radix Codonopsis (root of Codonopsis pilosula; Chinese name: dangshen) $[7,8]$, approved by the State Food and Drug Administration of the People's Republic of China in 1999 primarily as an antitumor injection to be manufactured and marketed in China $[9,10]$. Currently, there are many published trials about Shenqi Fuzheng Injection(SFI) combined with platinum-based chemotherapy for treatment of advanced NSCLC, some of which have shown that SFI may play an important role in the treatment of advanced NSCLC, could improve tumor response, performance status and reduce the toxicity of standard platinum-based chemotherapy. However, little is known about it outside of China, and there has not been a systematic evaluation until now. This paper presents a systematic review in an effort to clarify whether SFI in combination with platinum-based chemotherapy for advanced NSCLC really increases the efficacy and decreases the toxicity.

\section{Methods}

\section{Search strategy}

According to guidelines from the Cochrane collaboration [11], PubMed (1966 to April 2010); Cochrane Library (1988 to April 2010); EMBASE (1974 to April 2010); and Cochrane Central Register of Controlled Trials (1966 to April 2010); CBM (1978 to April 2010); CNKI(1984 to April 2010) were organized for search, and the following keywords were used: non-small-cell lung cancer, platinum-based chemotherapy, Shenqi Fuzheng injection, randomized controlled trials and multiple synonyms for each term. The publication languages were restricted to Chinese and English.

\section{Studies selection}

Trials were included if they were randomized controlled trials comparing a SFI plus platinum-based chemotherapy treatment group with a platinum-based chemotherapy control group for patients with advanced NSCLC.
Moreover, the reported data must have at least one of following outcomes: objective tumor response (the 4-point WHO scale [12] was adopted), performance status (the Karnofsky performance scale [13] was used and performance status was divided into 3 grades using a 10 -point change as the cutoff), and toxicity (the 5-point WHO scale [12] was used), and the reported data also needed to have sufficient detail to permit the calculation of the risk ratios and it's $95 \%$ CIs for each outcome. Data expressed as medians were not included in this meta-analysis, and the duplicates, case series, and case reports were also excluded.

\section{Data extraction}

All data on patient characteristics, treatment details, and clinical outcomes were independently abstracted and duplicated by two investigators (Ju Dong, Shi-Yue Su) using a standardized data collection form. Disagreements on study inclusion or data extraction were resolved by consensus of all coauthors. The outcome measures extracted were: objective tumor response, improved or stabilized performance status, and severe chemotherapy toxicity.

\section{Statistical analysis}

Meta-analysis was done with Review Manager 4.2 (The Cochrane Collaboration, Oxford, UK) [11]. Relative ratio (RR) and $95 \%$ confidence intervals $(\mathrm{CI})$ were calculated, hypothesis of homogeneity was not rejected, the fixed-effects model was used to calculate the summary relative ratio (RR), and the $95 \%$ CI. Otherwise, a random-effects model was used [14]. In this meta-analysis, three kind of following outcomes were calculated and analyzed appropriately.

\section{Objective tumor response}

The rate of tumor response was calculated as the number of patients experiencing complete response and partial response divided by the total number of patients (complete response plus partial response plus no change plus progressive disease) in each group, The RR of tumor response was calculated as the rate of tumor response in the SFI combined with platinum-based chemotherapy treatment group divided by that in the platinum-based chemotherapy control group. Thus, a RR of more than 1 favors the SFI combined with platinum-based chemotherapy treatment group. This method has been recommended by Sutton et al [15].

\section{Improved or stable performance status}

This is similar to the approach of Michael et al [5]. The rate of improved or stable performance status was calculated as the proportion of improved or stable performance status ( $>10$-point increase plus no change) divided by the total ( $>10$-point increase, plus no change, plus $>10$-point decrease). The RR of improved or stable 
performance status was analyzed as the rate of improved or stable performance status in the SFI combined with platinum-based chemotherapy treatment group, divided by this proportion in the platinum-based chemotherapy control group. Thus, a RR of more than 1 favors the SFI combined with platinum-based chemotherapy treatment group.

\section{Severe chemotherapy toxicity}

Using the approach of Delbaldo et al [16], the rate of severe chemotherapy toxicity was defined as the number of patients experiencing severe toxicity (WHO grades 3 and 4) divided by the total number of patients (WHO grades $0,1,2,3$ and 4) in each group. The RR of severe chemotherapy toxicity was analyzed as the proportion of severe toxicity in the SFI combined with platinum-based chemotherapy treatment group divided by this proportion in the platinum-based chemotherapy control group. Thus, a RR of less than 1 favors the SFI combined with platinum-based chemotherapy treatment group.

\section{Study quality evaluation}

Two reviewers (Ju Dong, Shi-Yue Su) independently graded each RCT/CCT using the modified Jadad scale[17]. The modified Jadad scale is an eight-item scale designed to assess randomization, blinding, withdrawals/dropouts, inclusion/exclusion criteria, adverse effects, and statistical analysis (table 1). The score for each article can range from 0 (lowest quality) to 8 (highest quality). Scores of 4-8 represent good to excellent (high quality) and 0 to 3 poor or low quality.

\section{Sensitivity analysis}

Sensitivity analysis was used to assess how robust the results are to uncertain decisions or assumption about the data and the methods that were used [18]. To analyze the sensitivity of our study, some studies were excluded because they were of low quality (had a quality score of 3 or under 3) and thus may weaken the conclusions.

\section{Publication bias analysis}

For the purposes of assessing the publication bias of this study, a funnel plot based on studies with data on objective tumor response (as this was the outcome with most studies included in meta-analysis) was graphed and Egger's test[19] was also performed.

\section{Results}

\section{Study characteristics and quality}

Twenty nine studies [20-48] were included in this review based on our selection criteria, encompassing 2,062 patients. A total of thirty studies were excluded due to lack of inclusion criteria, missing data and multiple publications. All included trials were published after 2004, and vinorelbine plus cisplatin (NP) was the most common chemotherapy regimen $(19 / 29,65.5 \%)$, and the remainder included paclitaxel plus cisplatin (TP), gemcitabine plus cisplatin (GP), and docetaxel plus cisplatin (DC). Of the 29 trials included in meta-analysis, 24 trials were reported as RCTs, and 5 trials didn't describe clearly the methods of grouping. Of the 24 trials claimed to be RCTs, the randomization procedure was described clearly and was true in only 5 trials(random digital table was adopted), 15 trials stated that subjects were "randomized" without describing the randomization method or procedures, 4 trials stated that methods that were not truly randomized were used. According to the modified Jadad scale, 10 studies were of high quality, with a quality score of 4 or above 4 , and the rest were of low quality, with a quality score of 3 or under 3 . Characteristics and quality of all included studies are presented in table 2.

The result of meta-analysis for Objective tumor response In the 29 included trials, the objective tumor response was reported by 27 trials [21-36,38-48], which included 1,849 patients. Meta-analysis showed there was a statistically significant higher tumor response rate (RR, 1.19; 95\% CI, 1.07 to 1.32; $\mathrm{P}=0.001$; Figure 1 ) in the SFI combined with platinum-based chemotherapy treatment group compared with the platinum-based chemotherapy control group, which meant the significant 19\% increase in the RR for the response rate was attributable to the SFI combined with platinum-based chemotherapy treatment group. Because heterogeneity may not lie in the different studies $(P=0.98)$ in this meta-analysis, the fixed-effect model was used.

\section{The result of meta-analysis for Performance status}

The rates of improved or stable performance status were reported in 20 trials $[20,21,23,25,26,28,30,31,33,36$ 43,45-47], which included 1336 patients. Meta-analysis showed there was a statistically significant higher rate of improved or stable performance status (RR, 1.57; 95\% CI, 1.45 to 1.70; P < 0.00001; Figure 2) when the SFI combined with platinum-based chemotherapy treatment group was compared with the platinum-based chemotherapy control group, which meant the significant $57 \%$ increase in the RR for the rate of improved or stable performance status was attributable to the SFI combined with platinum-based chemotherapy treatment group. For the same reason as objective tumor response, the fixed-effect model was performed in this metaanalysis.

\section{The result of meta-analysis for grade 3 or 4 WBC, PLT, HB, Nausea and Vomiting Toxicity}

In all included studies, 20 trials [20-25,27-29,32,34-36, $38,40-42,44,45,48]$ reported the number of patients with 
Table 1 The modified Jadad scale

\begin{tabular}{|c|c|c|}
\hline Eight-item of the modified Jadad scale & & Score \\
\hline \multirow[t]{2}{*}{ Was the study described as randomized? } & Yes & +1 \\
\hline & No & 0 \\
\hline \multirow[t]{3}{*}{ Was the method of randomization appropriate? } & Yes & +1 \\
\hline & No & -1 \\
\hline & Not described & 0 \\
\hline \multirow[t]{2}{*}{ Was the study described as blinding? ${ }^{a}$} & Yes & +1 \\
\hline & No & 0 \\
\hline \multirow[t]{3}{*}{ Was the method of blinding appropriate? } & Yes & +1 \\
\hline & No & -1 \\
\hline & Not described & 0 \\
\hline \multirow[t]{2}{*}{ Was there a description of withdrawals and dropouts? } & Yes & +1 \\
\hline & No & 0 \\
\hline \multirow[t]{2}{*}{ Was there a clear description of the inclusion/exclusion criteria? } & Yes & +1 \\
\hline & No & 0 \\
\hline \multirow[t]{2}{*}{ Was the method used to assess adverse effects described? } & Yes & +1 \\
\hline & No & 0 \\
\hline \multirow[t]{2}{*}{ Was the methods of statistical analysis described? } & Yes & +1 \\
\hline & No & 0 \\
\hline
\end{tabular}

a: double-blind got 1 score, single-blind got 0.5 score.

grade 3 or 4 white blood cell (WBC) toxicity, 18 trials [20-25,27-29,32,34-36,40-42,44,45] reported the number of patients with grade 3 or 4 platelet (PLT) toxicity, 15 trials $[20,22-25,28,29,32,34-36,41,42,44,45]$ reported the number of patients with grade 3 or 4 hemoglobin (HB) toxicity and 14 trials $[20,22-24,27-29,35,36,38,40-42,45]$ reported the number of patients with grade 3 or 4 nausea and vomiting. The rate of severe chemotherapy toxicity was calculated for WBC, PLT, HB, nausea and vomiting, and then meta-analyses were performed. As shown in Figures, the results indicated there was statistically significant lower severe toxicity for WBC (RR, 0.37 ; $95 \% \mathrm{CI}$, 0.29 to 0.47 ; $\mathrm{P}<0.00001$; Figure 3), PLT (RR, 0.33; 95\% CI, 0.21 to 0.52; $\mathrm{P}<0.00001$; Figure 4), HB (RR, 0.44; 95\% CI, 0.30 to 0.66; $\mathrm{P}<0.0001$; Figure 5) and nausea and vomiting (RR, 0.32; 95\% CI, 0.22 to 0.47 ; $\mathrm{P}<$ 0.00001; Figure 6) when the SFI plus platinum-based chemotherapy treatment group was compared with the platinum-based chemotherapy control group.

\section{The result of sensitivity analysis}

The sensitivity analysis revealed that with low quality studies excluded, the summary RR and $95 \%$ CIs for above outcomes were still similar to the results before they were excluded (table 3), which indicates that the results of our study are reliable and believable.

\section{The result of publication bias analysis}

Figure 7 is the funnel plot based on studies with data on objective tumor response, which is asymmetrical, and indicates that publication bias may have existed in our study. The result of Egger's test also suggested an evidence of publication bias $(P=0.016)$.

\section{Discussion}

In medicine, systematic reviews and meta-analysis form the core of a movement to ensure that medical treatments are based on the best available empirical data. One important advantage for meta-analysis is that it can enable the user to perform statistical synthesis and then it can be used to enhance the statistical power to obtain a more accurate conclusion [49]. Thus, to systematically evaluate whether SFI increases the efficacy and decreases the toxicity when combined with platinum-based chemotherapy for advanced NSCLC, the authors conducted a systematic review. The results suggested that SFI intervention may enhance tumor response, improve performance status, and reduce chemotherapy toxicity, when compared with platinum-based chemotherapy alone. This is the first systematic review of SFI for advanced NSCLC and the results can provide important references about how to reduce toxicity and enhance the curative effect of platinum-based chemotherapy. In China, it is common to use SFI to treat advanced NSCLC, but no relevant articles or evaluations have been published in the English medical journals, hence reducing its worldwide validity. This study may prove useful for supplementing the evidence for the use of SFI in the treatment of advanced NSCLC.

Shenqi Fuzheng Injection is concocted from Radix Astragali(huangqi) and Radix Codonopsis(dangshen). These two kind of Chinese medicinal herbs have been 
Table 2 Study characteristics and quality

\begin{tabular}{|c|c|c|c|c|c|c|}
\hline \multirow[t]{2}{*}{ Studies (Author) } & \multirow[t]{2}{*}{ Year } & \multicolumn{2}{|c|}{$\begin{array}{l}\text { Chemotherapy } \\
\text { regimen }^{\mathrm{a}}\end{array}$} & \multirow[t]{2}{*}{ Number $(T / C)$} & \multirow[t]{2}{*}{ Type of Assessable Outcomes } & \multirow[t]{2}{*}{ Jadad Scores $^{\mathrm{b}}$} \\
\hline & & treatment & control & & & \\
\hline Hao XL[20] & 2008 & $\mathrm{NP}+\mathrm{SFI}$ & NP & $60 / 68$ & WBC/HB/PLT/nausea and vomiting toxicity, KPS & 3 \\
\hline Wang K[21] & 2007 & $\mathrm{NP}+\mathrm{SFI}$ & NP & $18 / 18$ & tumor response, WBC/PLT toxicity, KPS & 3 \\
\hline Kang GY[22] & 2006 & $\mathrm{NP}+\mathrm{SFI}$ & NP & $36 / 36$ & tumor response, WBC/PLT/HB/nausea and vomiting toxicity, KPS & 2 \\
\hline Gong ZM[23] & 2008 & $\mathrm{NP}+\mathrm{SFI}$ & NP & $33 / 32$ & tumor response, WBC/PLT/HB/nausea and vomiting toxicity, KPS & 2 \\
\hline Wang XY[24] & 2007 & $\mathrm{NP}+\mathrm{SFI}$ & NP & $35 / 34$ & tumor response, WBC/PLT/HB/nausea and vomiting toxicity, & 4 \\
\hline Wang YZ[25] & 2007 & $\mathrm{NP}+\mathrm{SFI}$ & NP & $28 / 27$ & tumor response, KPS, WBC/PLT/HB toxicity & 3 \\
\hline Li TW[26] & 2009 & $\mathrm{NP}+\mathrm{SFl}$ & NP & $36 / 33$ & tumor response, the KPS & 4 \\
\hline Li Y[27] & 2007 & $\mathrm{NP}+\mathrm{SFI}$ & NP & $44 / 43$ & tumor response, WBC/PLT/nausea and vomiting toxicity, & 4 \\
\hline LV J[28] & 2008 & $\mathrm{NP}+\mathrm{SFI}$ & NP & $40 / 40$ & tumor response, WBC/PLT/HB/nausea and vomiting toxicity, KPS & 4 \\
\hline Zhao ZY[29] & 2007 & $\mathrm{NP}+\mathrm{SFI}$ & NP & $35 / 34$ & tumor response, WBC/PLT/HB nausea and vomiting toxicity, & 4 \\
\hline Geng L[30] & 2004 & $\mathrm{NP}+\mathrm{SFI}$ & NP & $25 / 15$ & tumor response, KPS & 2 \\
\hline Yu QZ[31] & 2007 & $\mathrm{NP}+\mathrm{SFI}$ & NP & $30 / 32$ & tumor response, KPS & 4 \\
\hline Liu CL[32] & 2004 & $\mathrm{NP}+\mathrm{SFI}$ & NP & $60 / 60$ & tumor response, WBC/PLT/HB toxicity & 2 \\
\hline Liu PH[33] & 2007 & $\mathrm{NP}+\mathrm{SFI}$ & NP & $30 / 30$ & tumor response, KPS & 1 \\
\hline Pan YK[34] & 2008 & $\mathrm{NP}+\mathrm{SFI}$ & NP & $45 / 45$ & tumor response, WBC/PLT/HB toxicity & 2 \\
\hline Zheng $J H[35]$ & 2009 & $\mathrm{NP}+\mathrm{SFI}$ & NP & $42 / 42$ & tumor response, WBC/PLT/HB/nausea and vomiting toxicity & 4 \\
\hline Miao SR[36] & 2010 & $\mathrm{NP}+\mathrm{SFI}$ & NP & $38 / 41$ & tumor response, the KPS, WBC/PLT/nausea and vomiting toxicity & 3 \\
\hline Li YQ[37] & 2010 & $\mathrm{NP}+\mathrm{SFI}$ & NP & $43 / 42$ & KPS & 5 \\
\hline Geng D[38] & 2007 & $\mathrm{NP}+\mathrm{SFI}$ & NP & $42 / 26$ & tumor response, WBC/the nausea and vomiting toxicity & 2 \\
\hline Zou Y[39] & 2005 & $\mathrm{TP}+\mathrm{SFI}$ & $\mathrm{TP}$ & $24 / 24$ & tumor response, KPS & 3 \\
\hline Luo SZ[40] & 2006 & $\mathrm{TP}+\mathrm{SFI}$ & TP & $25 / 25$ & tumor response, KPS, WBC/PLT/nausea and vomiting toxicity & 2 \\
\hline Luo SW[41] & 2007 & $\mathrm{TP}+\mathrm{SFI}$ & TP & $30 / 30$ & tumor response, WBC/PLT/HB/nausea and vomiting toxicity, KPS & 2 \\
\hline Zhang FL[42] & 2008 & $\mathrm{TP}+\mathrm{SFI}$ & TP & $30 / 30$ & tumor response, WBC/PLT/HB/nausea and vomiting toxicity, KPS & 3 \\
\hline Zhao YX[43] & 2009 & $\mathrm{TP}+\mathrm{SFI}$ & $\mathrm{TP}$ & $40 / 40$ & tumor response, KPS & 2 \\
\hline Yu F[44] & 2007 & $\mathrm{DC}+\mathrm{SFI}$ & DC & $30 / 30$ & tumor response, WBC/PLT/HB toxicity & 4 \\
\hline He WJ[45] & 2008 & $\mathrm{GP}+\mathrm{SFI}$ & GP & $35 / 35$ & tumor response, WBC/PLT/HB/nausea and vomiting toxicity, KPS & 3 \\
\hline Liang K[46] & 2010 & $\mathrm{GP}+\mathrm{SFI}$ & GP & $39 / 37$ & tumor response, KPS, & 2 \\
\hline Chen J[47] & 2007 & $\mathrm{TP} / \mathrm{NP}+\mathrm{SFI}$ & $\mathrm{TP} / \mathrm{NP}$ & $41 / 39$ & tumor response, KPS & 2 \\
\hline Wu L[48] & 2004 & $\mathrm{TP} / \mathrm{NP}+\mathrm{SFI}$ & TP/NP & $30 / 30$ & tumor response, WBC toxicity & 5 \\
\hline
\end{tabular}

Abbreviations: SFI, shenqi fuzheng injection; NP, vinorelbine, cisplatin; TP, paclitaxel, carboplatin; DC, docetaxel cisplatin; GP, gemcitabine cisplatin;T/C, treatment group/control group; KPS, Karnofsky Performance status; WBC, white blood cell; PLT, platelet; HB, hemoglobin;

a: all patients included in studies in both groups received systemic chemotherapy therapy, and no patients received surgery and radiation, The only difference between the two groups was whether they received SFI.

b: modified Jadad scale was used.

used in China and some other Asia countries as herbal medicines for many years. Of them, Radix Astragali is usually used as an immunomodulating agent in the treatment of immunodeficiency diseases and to alleviate the adverse effects of chemotherapeutic drugs $[50,51]$. Radix Codonopsis is usually used to treat dyspepsia, fatigue, bronchitis, cough, inflammation and so on, and its pharmacological activities such as antifatigue and immunomodulatory activities were also reported[52]. SFI is developed from Radix Astragali and Codonopsis, which suggests that its effect in the treatment of NSCLC may be related with the above pharmacological activities of Radix Astragali and Codonopsis. However, what are the specific immunological and cytotoxic mechanisms? what are main effective components? Do the interactions between medicines or components exist? These questions are not clear and require further investigation.

This systematic review also has limitations. First, allocation concealment and blinding were not described in all included trials, which may result in the emergence of bias, and the overestimation of the efficacy of the treatment group. Second, much of the data on the patients' survival was not reported in the included studies, thus the influence that SFI combined with platinum-based chemotherapy had on survival could not be analyzed by this systematic review. Third, funnel plot and Egger's test suggested publication bias may exist. Given above reasons, the evidence from this study may be insufficient, and should be carefully disseminated to the medical 


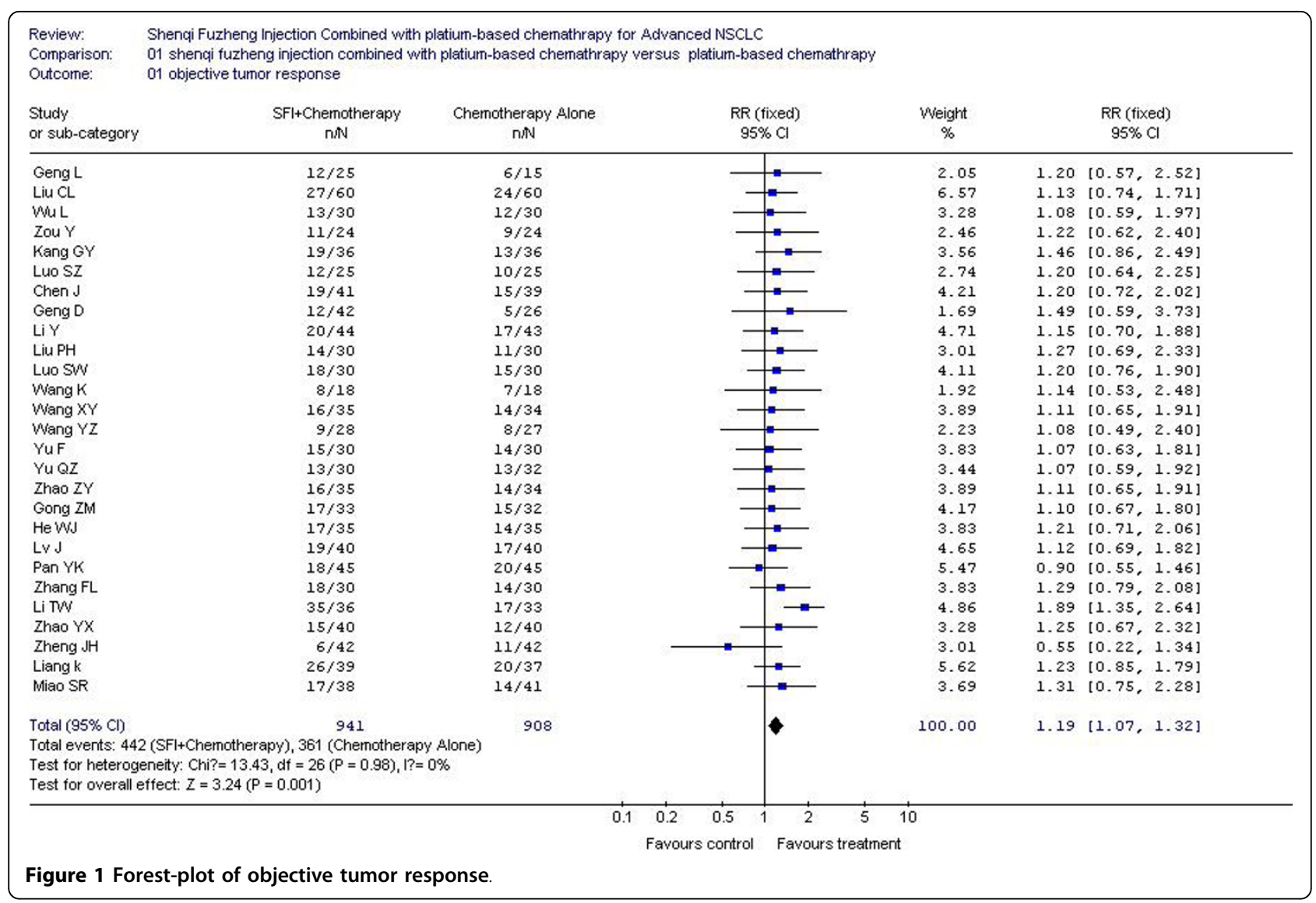

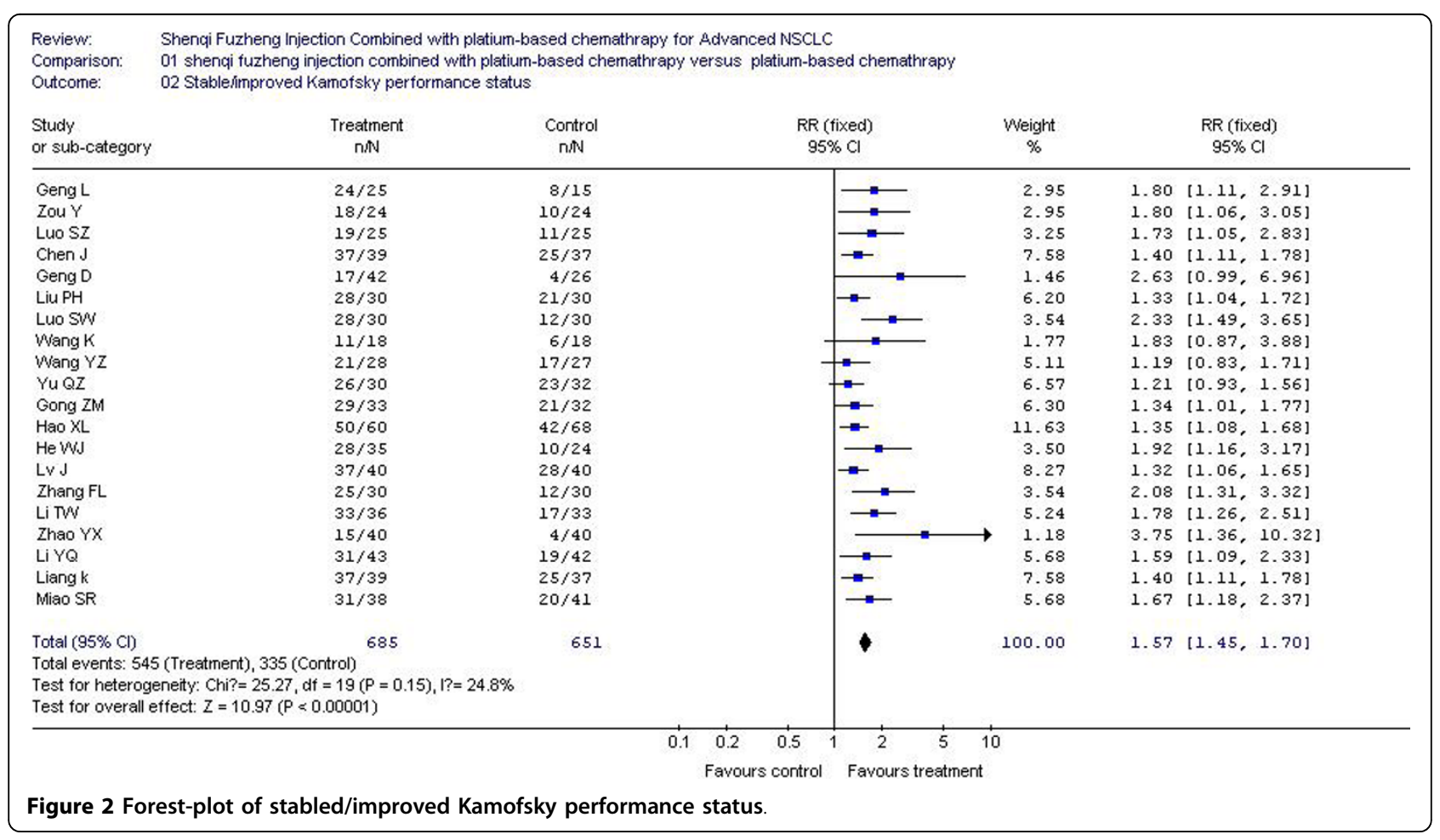




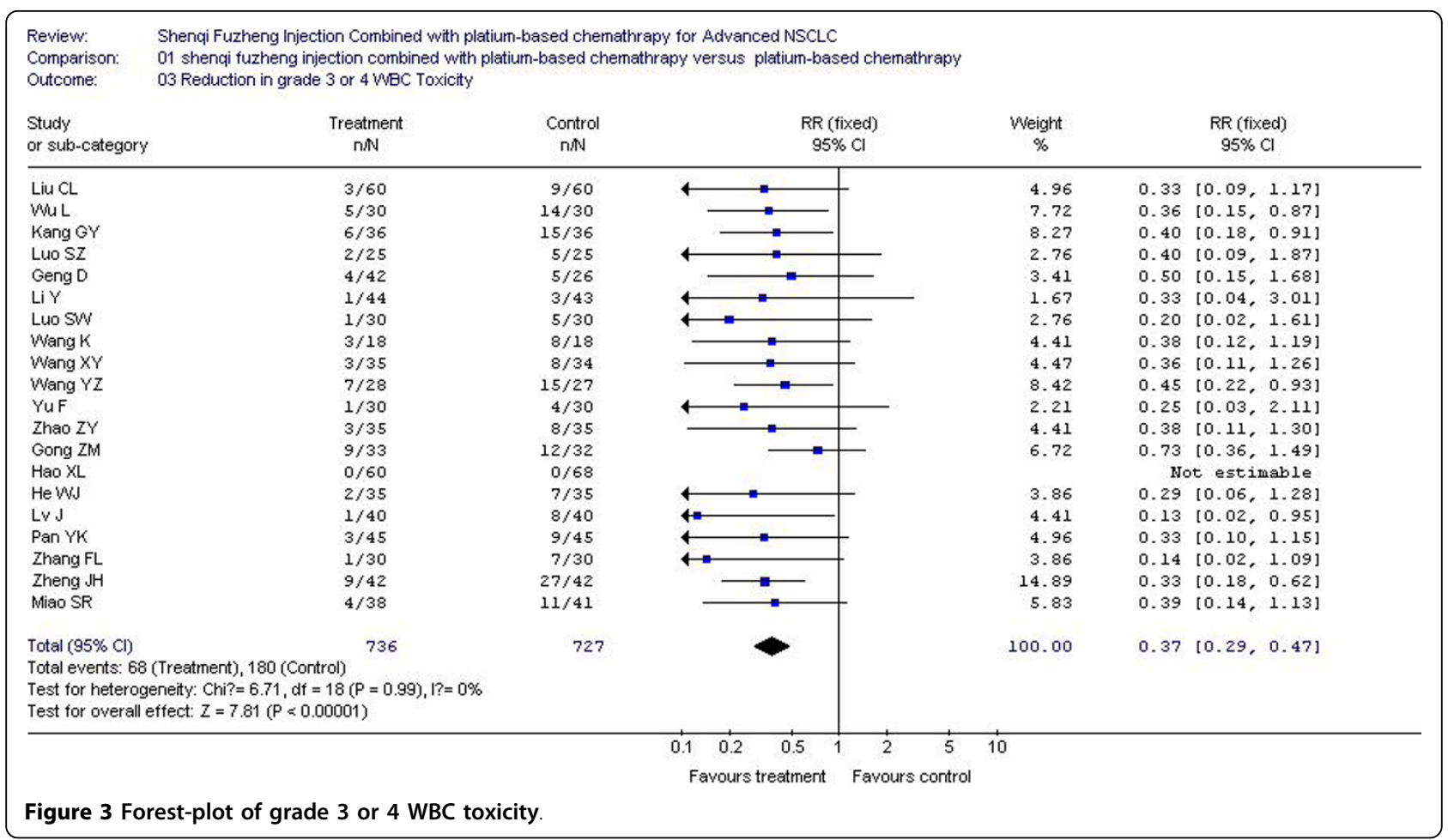

community. However, we all know it is difficult and expensive to carry out clinical trials on advanced NSCLC patients and large, placebo-controlled, double-blind studies are almost impossible. Therefore, trials with above questions may exist in many countries and may be permitted to some extent, but still provide helpful information for clinical practice and drug development. Now it has been increasingly recognized that Western medicine may not be the answer for the treatment of all diseases and sometimes alternative medicines or treatment

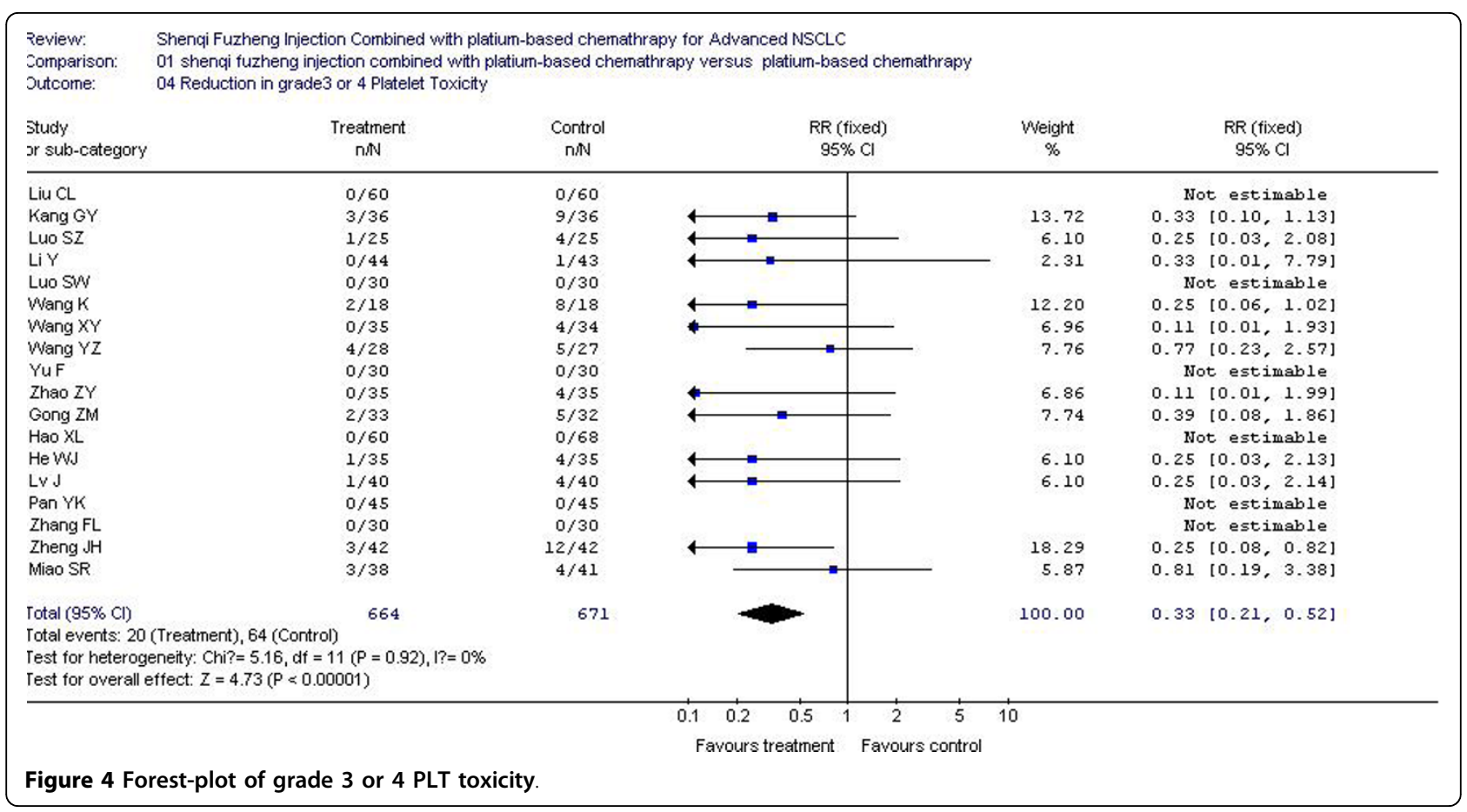




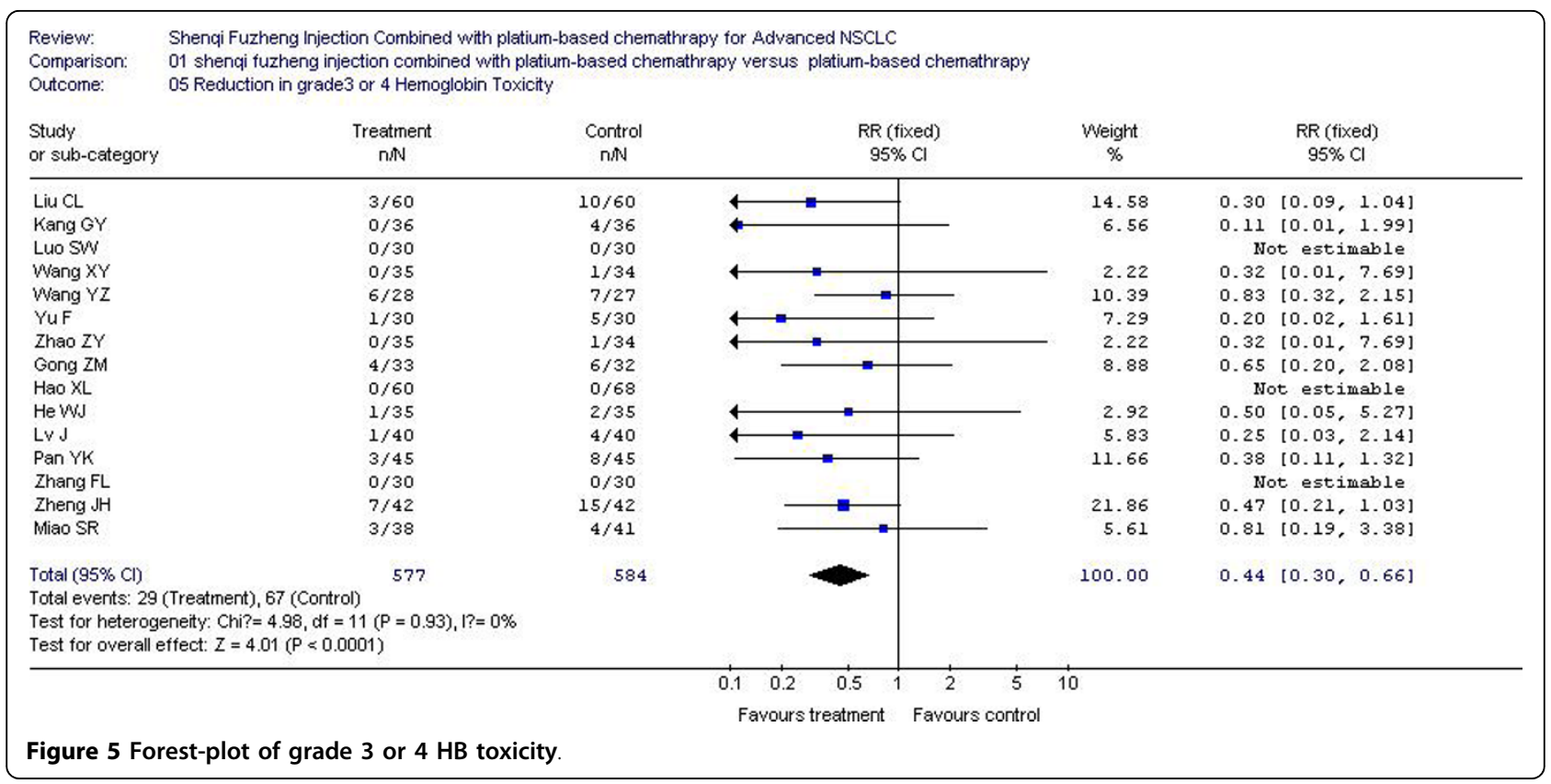

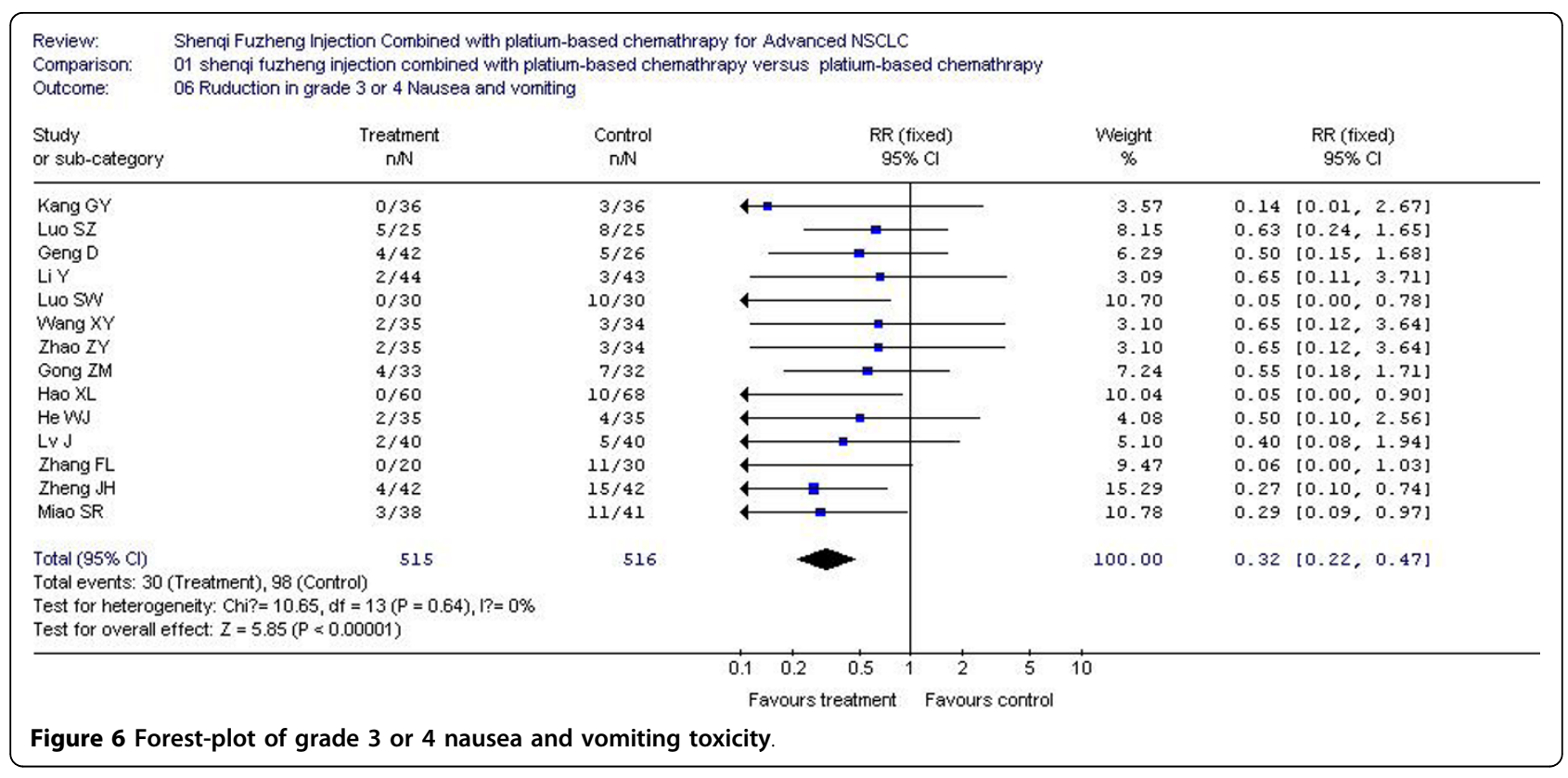

regimes may prove successful. Therefore, though SFI is a kind of traditional Chinese medicine, the results of this systematic review suggested it may play an important role in the treatment of advanced NSCLC.

\section{Conclusions}

In conclusion, in this systematic review evidence was found that SFI intervention may increase the efficacy and reduce the toxicity when combined with platinumbased chemotherapy for advanced NSCLC, which would provide important references about how to reduce toxicity and enhance the curative effect of platinum-based chemotherapy for advanced NSCLC. However, limitations remain and the results needs to be further verified by more high-quality trials.

\section{Acknowledgements}

This study was supported by a postgraduate innovation project from Jiangsu Province Education Department, and also supported by National Natural

Science Foundation of China (No.30973715). The authors are grateful to the 
Table 3 Sensitivity analysis of this study

\begin{tabular}{ccccccccc}
\hline Outcomes & \multicolumn{3}{c}{ All Studies } & \multicolumn{3}{c}{ Good Quality Studies } \\
\cline { 2 - 8 } & N & Patients & RR $(\mathbf{9 5} \% \mathbf{C l})$ & $\mathbf{P}$ & $\mathbf{N}$ & Patients & RR (95\%Cl) & P \\
\hline Tumor response & 27 & 1849 & $1.19[1.07,1.32]$ & 0.001 & 9 & 640 & $1.16[0.98,1.38]$ & 0.08 \\
KPS & 20 & 1336 & $1.57[1.45,1.70]$ & $<0.00001$ & 4 & 296 & $1.45[1.25,1.68]$ & $<0.00001$ \\
WBC & 20 & 1463 & $0.37[0.29,0.47]$ & $<0.00001$ & 7 & 510 & $0.32[0.21,0.48]$ & $<0.00001$ \\
PLT & 18 & 1335 & $0.33[0.21,0.52]$ & $<0.00001$ & 6 & 450 & $0.21[0.09,0.50]$ & 0.0005 \\
HB & 15 & 1161 & $0.44[0.30,0.66]$ & $<0.001$ & 5 & 362 & $0.37[0.19,0.72]$ & 0.003 \\
Nausea and Vomiting & 14 & 1031 & $0.32[0.22,0.47]$ & $<0.00001$ & 5 & 389 & $0.41[0.22,0.77]$ & 0.006 \\
\hline
\end{tabular}

Abbreviations: KPS, Karnofsky Performance status; WBC, white blood cell; PLT, platelet; HB, hemoglobin; $\mathrm{N}$, the number of trials.

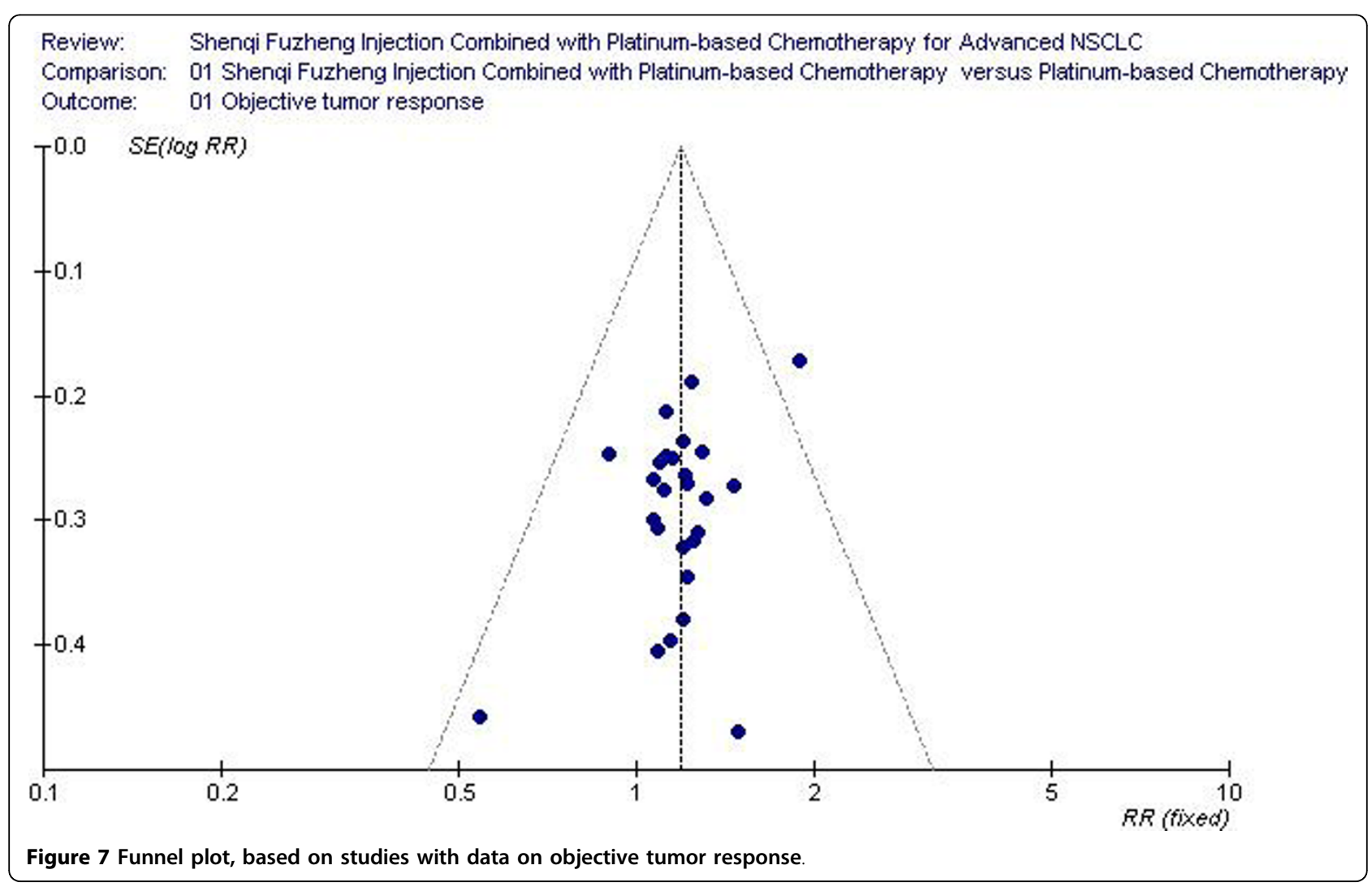

help of Prof Xiu-Lin Gong in writing, and the authors also appreciate the editor board and the reviewers for their work on this paper.

\section{Author details}

${ }^{1}$ Institute of Basic Medical Science, Nanjing University of Traditional Chinese Medicine, Nanjing, P.R China. ${ }^{2}$ Chuzhou City People's Hospital The Second Party, Chuzhou, P.R China. ${ }^{3}$ Institute of Basic Medical Science, Nanjing University of Traditional Chinese Medicine, Nanjing, P.R China. ${ }^{4}$ Institute of Basic Medical Science, Nanjing University of Traditional Chinese Medicine, Nanjing, P.R China.

\section{Authors' contributions}

JD, ZZ conceived the study, JD, SYS, MYW, ZZ participated in protocol design. JD, SYS ran the searches and abstracted data. JD performed the analysis. JD, SYS, MYW, ZZ wrote and approved the manuscript.

\section{Competing interests}

The authors declare that they have no competing interests.
Received: 23 August 2010 Accepted: 22 October 2010 Published: 22 October 2010

\section{References}

1. Molina JR, Yang P, Cassivi SD, Schild SE, Adjei AA: Non-small cell lung cancer: epidemiology risk factors, treatment, and survivorship. Mayo Clin Proc 2008, 83(5):584-594.

2. Suresh R, Chandra P: Systemic chemotherapy for advanced non-small cell lung cancer: recent advances and future directions. The Oncologist 2008, 3(suppl 1):5-12.

3. Barlesi F, Pujol JL, Daures J-P: Should chemotherapy $(C x)$ for advanced non-small cell lung cancer (NSCLC) be platinum-based? a literaturebased meta-analysis of randomized trials. J Clin Oncol 2005, 23(16s):673s.

4. D'Addario G, Pintilie M, Leighl NB, Fied R, Gerny T, Shepherd FA: Platinumbased versus non platinum-based chemotherapy in advanced nonsmall-cell lung cancer: a meta-analysis of the published literature. J Clin Oncol 2005, 23(13):2926-2936.

5. McCulloch M, See C, Shu XJ, Broffman M, Kramer A, Fan WY, Gao J, Lieb W, Shieh K, Colford JM Jr: Astragalus-based chinese herbs and platinum- 
based chemotherapy for advanced non small cell lung cancer: metaAnalysis of randomized trials. J Clin Oncol 2006, 224(3):419-430.

6. Wu BC, Xu L, Chen M: Meta-analysis of Ai Di injection combined with NP chemotherapy in the treatment of late stage non-small cell lung cancer. Zhejiang Journal of Integrated Traditional Chinese and Western Medicine 2009, 19(7):446-447.

7. Yang LR, Xu XY: Clinical application and curative effect evaluation of Shenqi fuzheng injection. Heilongjiang Journal of Traditional Chinese Medicine 2004, 4:46-48

8. Lu Y, Lu YX: Clinical application and pharmacology function of Shenqi fuzheng injection. Li Shi Zhen Medicine and material medica research 2006 17(10):2083-2085.

9. Pan L: Practical road of "the numeral turn Chinese herbal medicine" for Shenqi fuzheng injection. Journal of China Prescription Drug 2009, 1(82):37-39.

10. Zhong ZH: Dictate history: A national new medicine that come from experiential prescription. Journal of China Prescription Drug 2009, 1(82):33-36.

11. Higgins J, Green S, (eds): Cochrane handbook for systematic reviews of interventions 4.2.6. In the Cochrane library Wiley: Chichester 2006, 301(4), (updated September 2006).

12. WHO: WHO Handbook for Reporting Results of Cancer Treatment Geneva, Switzerland, World Health Organization 1979.

13. Yates JW, Chalmer B, McKegney FP: Evaluation of patients with advanced cancer using the Karnofsky performance status. Cancer 1980, 45:2220-2224.

14. DerSimonian R, Laird N: Meta-analysis in clinical trials. Control Clin Trials 1986, 7:177-188.

15. Sutton AJ, Abrams KR, Jones DR, Sheldon TA, Song F: Methods for MetaAnalysis in Medical Research Chichester, United Kingdom, John Wiley \& Sons 2000.

16. Delbaldo C, Michiels S, Syz N: Benefits of adding a drug to a single-agent or a 2-agent chemotherapy regimen in advanced non-small-cell lung cancer: A meta-analysis. JAMA 2004, 292:470-484.

17. Oremus M, Wolfson C, Perrault A, Demers L, Momoli F, Moride Y: Interrater reliability of the modified Jadad quality scale for systematic reviews of Alzheimer's disease drug trials. Dement Geriatr Cogn Disord 2001, 12:232-236.

18. Breierova L, Choudhari M: An introduction to sensitivity analysis. MIT Pr 1996, 41-107.

19. Egger M, Davey SG, Altman DG: Systematic reviews in health care: Metaanalysis in context. London: BMJ books 2001.

20. Hao $X L, L v X J$ : The influence of Shenqi fuzheng injection combined with chemotherapy on the survival quality of late-stage non-small cell lung cancer. Chinese Journal of Practical oncology 2008, 22(5):458-459.

21. Wang $K$, Tan JX, Nong Y: Clinical observation of Shenqi fuzheng injection combined with NP chemotherapy in the treatment of late stage nonsmall cell lung cancer. Modern Journal of Integrated Traditional Chinese and Western Medicine 2007, 16(26):3797-3798.

22. Kang GY, Li B: Shenqi fuzheng injection combined with chemotherapy for the late stage non-small cell lung cancer 36 cases. Chinese Journal of Integrative Medicine 2006, 26(6):565-566.

23. Gong ZM, Wang Y, Yu CY, Chen HY: Clinical observation of Shenqi fuzheng injection combined with NP chemotherapy for the elder latestage non-small cell lung cancer. Information on Traditional Chinese Medicine 2008, 15(9):64-65.

24. Wang XY, Hang ZQ, Li H, Cai CB: Clinical observation and nursing of Shenqi fuzheng injection combined with NP chemotherapy for the elder late-stage non-small cell lung cancer. Journal of Chinese Lung Cancer 2007, 10(3):234-236.

25. Wang YZ, Yang ZX, Liao SH, Shen X: Clinical observation of Shenqi fuzheng injection combined with vinorelbine plus carboplatinum for the late stage non-small cell lung cancer. Journal of Chinese clinical intern Medicine 2007, 24(3):206-207.

26. Li TW, Xiang L, Tong FY, Zhang CH: Clinical observation of Shenqi fuzheng injection combined with chemotherapy for the late stage lung cancer. Journal of Progress in Modern Biomedicine 2009, 9(10):1917-1919.

27. Li Y, Chen SX, Huang RW: Clinical observation of Shenqi fuzheng injection combined with NP chemotherapy for the late stage non-small cell lung cancer. Journal of Chinese Modern Medicine 2007, 9(3):40-41.
28. LV J: Clinical observation of Shenqi fuzheng injection combined with chemotherapy for the late stage non-small cell lung cancer. China Medical Herald 2008, 5(36):73-74.

29. Zhao ZY, Wu DL, Chen M, Jiang H, Yan GJ: The short-term curative effect of Shenqi fuzheng injection combined with NP chemotherapy for the elder late stage non-small cell lung cancer. Journal of Chinese modern oncology 2007, 15(1):42-43.

30. Geng L: Shenqi fuzheng injection combined with chemotherapy for the non-small cell lung cancer. Journal of Medical Forum 2004, 25(17):29-30.

31. Yu QZ: Shenqi fuzheng injection combined with chemotherapy for the middle and late stage non-small cell lung cancer. Chinese Journal of Integrative Medicine 2007, 27(5):473-474.

32. Liu CL, Chen WP, Cui SZ, Den GY, Liu LP, Tan LH, Su XC, Yan BC, Kong JX: Clinical observation of Shenqi fuzheng injection combined with chemotherapy for the elder non-small cell lung cancer. Chinese Journal of Integrative Medicine 2004, 24(10):901-903.

33. Liu FH, Liao GQ, Wang HM: The curative effect observation of Shenqi fuzheng injection combined with chemotherapy for the late stage lung cancer. Journal of Third Military Medical University 2007, 29(3):259-260.

34. Pan YK, Huang M, Qu M: Clinical observation of Shenqi fuzheng injection assisted with chemotherapy for the non-small cell lung cancer. Journal of Chinese Clinical Medical 2008, 3(4):43-45.

35. Zhen JH, Chen YF: Shenqi fuzheng injection combined with NP chemotherapy in treating elder late stage non-small cell lung cancer patients 42 cases. JiangXi Journal of Traditional Chinese Medicine 2009, 40(6):58-59.

36. Miao SR, Yang WH, Geng CH: Clinical observation of Shenqi fuzheng injection combined with NP chemotherapy in treating elder late stage non-small cell lung cancer. Chinese Journal of Practical Medicine 2010, 5(11):16-17.

37. Li YQ, Zhou X, Zhang T, Chen HJ: The clinical study on reducing toxicity effect of Shenqi fuzheng injection combined with NP chemotherapy for non-small cell lung cancer. Chinese Journal of New Drugs 2010, 19(2):23-126.

38. Geng D, Cui JC, Ma L: The curative effect observation of Shenqi fuzheng injection combined with NP chemotherapy for the late stage non-small cell lung cancer. Chinese Journal of Practical Medicine 2007, 2(5):57-59.

39. Zou Y, Bo YJ, Ruan PG: Clinical observation of Shenqi fuzheng injection combined with paclitaxel plus carboplatinum chemotherapy in treating patients with late stage non-small cell lung cancer. Chinese Journal of Practical oncology 2005, 20(3):260-262.

40. Luo SZ, Long JH, Yu XY: Clinical observation of Shenqi fuzheng injection combined with paclitaxel plus cisplatinum chemotherapy for late stage non-small cell lung. Journal of Chinese cancer research and clinic 2006, 18(3):181-183.

41. Luo SW, Huang YP, Shan HL, Yang YW, Mo C, Wu XE: Clinical observation of middle and late stage non-small cell lung cancer treated with Shenqi fuzheng injection combined with paclitaxel plus carboplatinum. Chinese Journal of Clinical Oncology 2007, 12(5):381-382.

42. Zhang FL: Clinical observation of middle and late stage non-small cell lung cancer treated with Shenqi fuzheng injection combined with paclitaxel plus carboplatinum. Journal of Chinese Modern Oncology 2008, 16(7):1165-1166

43. Zhao YX, Wang CY, Li J, Wang F: The curative effect observation of Shenqi fuzheng injection combined with paclitaxel plus carboplatinum for non-small cell lung cancer. Journal of Chinese misdiagnose 2009, 19(21):5129-5130.

44. Yu F, Li K: Clinical observation of Shenqi fuzheng injection assisted with chemotherapy for non-small cell lung cancer. Chinese Journal of Integrative Medicine 2007, 21(2):166-167.

45. He WJ, Zhao JQ: Clinical observation of Shenqi fuzheng injection combined with gemcitabine plus cisplatinum for late stage Non-small Cell Lung Cancer. Journal of Chinese progress in oncology 2008, 6(4):432-434

46. Liang K, Li SY: The curative effect observation of Shenqi fuzheng injection combined with chemotherapy for non-small cell lung cancer. Journal of Chinese Tropical Medicine 2010, 10(4):498-499.

47. Chen J, Jia YJ, Sun YY, Zhang YC: The clinical observation of Shenqi fuzheng injection combined with chemotherapy for non-small cell lung cancer. Chinese Medicine Emergency 2007, 16(8):911-912. 
48. Wu L, Jiang B, Yang J, Li H: Shenqi fuzheng injection combined with chemotherapy in treating elder late stage non-small cell lung cancer patients 30 cases. Chinese Journal of Integrative Medicine 2004, 24(6):567-568.

49. Michael Borenstein L, Hedges V, Higgins JPT, HR : Introduction to MetaAnalysis Rothstein@ John Wiley \& Sons, Ltd 2009.

50. Ma XQ, Shi Q, Duan JA, Dong TT, Tsim KW: Chemical analysis of Radix Astragali (Huangqi) in China: a comparison with its adulterants and seasonal variations. J Agric Food Chem 2002, 50:4861-4866.

51. Shao BM, Xu W, Dai H, Tu P, Li Z, Gao XM: A study on the immune receptors for polysaccharides from the roots of astragalus membranaceus, a chinese medicinal herb. Biochem Biophys Res Commun 2004, 320:1103-1111.

52. Jiao HJ: The pharmacology efficacy and clinical application about dangshen. Chinese Journal of Clinical Medicine 2005, 25(4):89-92.

doi:10.1186/1756-9966-29-137

Cite this article as: Dong et al: Shenqi fuzheng, an injection concocted from chinese medicinal herbs, combined with platinum-based chemotherapy for advanced non-small cell lung cancer: a systematic review. Journal of Experimental \& Clinical Cancer Research 2010 29:137.

\section{Submit your next manuscript to BioMed Central} and take full advantage of:

- Convenient online submission

- Thorough peer review

- No space constraints or color figure charges

- Immediate publication on acceptance

- Inclusion in PubMed, CAS, Scopus and Google Scholar

- Research which is freely available for redistribution

Submit your manuscript at www.biomedcentral.com/submit 